\title{
Health-Related Quality of Life in Non-Hodgkin Lymphoma Survivors: A Prospective Cohort Study
}

\section{Danbee Kang, $\mathrm{PhD}^{1}$ \\ Juhee Cho, $\mathrm{PhD}^{1,2}$ \\ Im Ryung Kim, MSN² \\ Mi Kyung Kim, RN ${ }^{3}$ \\ Won Seog Kim, MD, PhD ${ }^{4}$ \\ Seok Jin Kim, MD, PhD ${ }^{1,4}$}

\section{${ }^{1}$ Department of Health Sciences and Technology, SAIHST, Sungkyunkwan University, Seoul, ${ }^{2}$ Cancer Education Center, Samsung Comprehensive Cancer Center, Samsung Medical Center, Sungkyunkwan University School of Medicine, Seoul, ${ }^{3}$ Samsung Biomedical Research Institute, Samsung Medical Center, Seoul, ${ }^{4}$ Division of Hematology-Oncology, Department of Medicine, Samsung Medical Center, Sungkyunkwan University School of Medicine, Seoul, Korea}

\section{Purpose}

We evaluated health-related quality of life (HRQOL) in long-term survivors of indolent and aggressive non-Hodgkin lymphoma (NHL).

\section{Materials and Methods}

The HRQOL was assessed by the European Organization for Research and Treatment of Cancer Quality-of-Life Questionnaire Core 30 (EORTC QLQ-C30) at diagnosis in NHL patients between 2008 and 2011, and follow-up evaluation was conducted from June 2014 to February 2015 using EORTC QLQ-C30 and the quality of life in cancer survivors (QOL-CS) questionnaire. We used linear mixed models to compare changes in HRQOL between indolent and aggressive NHL over time.

\section{Results}

The HRQOL of long-term survivors with aggressive NHL improved to the similar level of indolent NHL during the follow-up survey. However, survivors of NHL were found to fear the probability of relapse and second malignancy, and the degree of fear was not different between survivors with aggressive stage I/II or III/IV NHL ( $p>0.05$ ). Furthermore, a half of survivors reported impaired sense of psychosocial well-being regardless of aggressiveness and stage during follow-up survey. More than $65 \%$ of survivors thought they did not receive sufficient support from others, and patients who had financial difficulties at diagnosis were more frequently associated with suffering from insufficient support. Impaired physical and cognitive functioning at diagnosis was significantly associated with lack of life purpose in long-term survivors.

\section{Conclusion}

The HRQOL of aggressive NHL survivors improved to a similar level to that of indolent NHL. However, the majority of survivors still had fear of relapse, and psychosocial well-being remained unmet needs. 81 Irwon-ro, Gangnam-gu, Seoul 06351, Korea Tel: 82-2-3410-1766

Fax: 82-2-3410-1754

E-mail: kstwoh@skku.edu

Key words

Quality of life, Distress, Psychosocial well-being, Non-Hodgkin lymphoma, Survivors

\section{Introduction}

Non-Hodgkin lymphoma (NHL) is a group of lymphoid malignant disorders accounting for the most common hematologic malignancies in Western and Asian countries. These disorders are clinically classified into aggressive and indolent
NHL according to clinical course and outcome $[1,2]$. As a whole, the survival outcomes of NHL patients have improved due to advances in treatment, including novel targeted drugs [3-5]. For example, the treatment outcome of patients with diffuse large B-cell lymphoma (DLBCL) has improved since introduction of rituximab, a monoclonal antibody targeting the CD20 antigen [6]. Nevertheless, 
patients tend to be seriously concerned about their disease once diagnosed with NHL, regardless of whether the aggressive or indolent form. After completing treatment, patients also commonly experience physical and psychological adverse events due to treatment-related toxicities. Problems such as functional impairment, fatigue, post-traumatic stress, and financial difficulties tend to be long term and impair the health-related quality of life (HRQOL) of NHL survivors [7-10]. Because the number of long-term survivors of NHL is continuously increasing, there is an increasing need for survivorship care among NHL survivors with impaired HRQOL $[9,11]$. Indeed, patients receiving treatments such as chemotherapy were reported to have impaired physical functioning and deteriorated psychological well-being in a previous study evaluating the HRQOL of lymphoma [12,13]. The ultimate goal of survivorship care is basically restoring the physical and psychological status of long-term cancer survivors to their previous healthy pre-diagnosis state. Therefore, changes in HRQOL from diagnosis to after treatment should be considered for appropriate management of longterm survivors. However, there are limited data comparing HRQOL between NHL diagnosis and survivorship $[9,14,15]$.
In addition, while aggressive NHL survivors are expected to have poor HRQOL due to various physical and psychological problems during treatment, previous studies did not compare HRQOL between aggressive and indolent NHL survivors. Thus, this study compares HRQOL at diagnosis to that of long-term follow-up among survivors of aggressive and indolent NHL. Furthermore, the specific psychological, social, and spiritual well-being of NHL survivors with high unmet needs was assessed.

\section{Materials and Methods}

\section{Study participants}

This is an add-on study of the Samsung Medical Center Lymphoma Cohort Study (SMC-LCS, NCT00822731) conducted from June 2014 to February 2015 at Samsung Medical Center, Seoul, Korea. The SMC-LCS recruited patients diagnosed with Hodgkin and NHL from September 2008 to

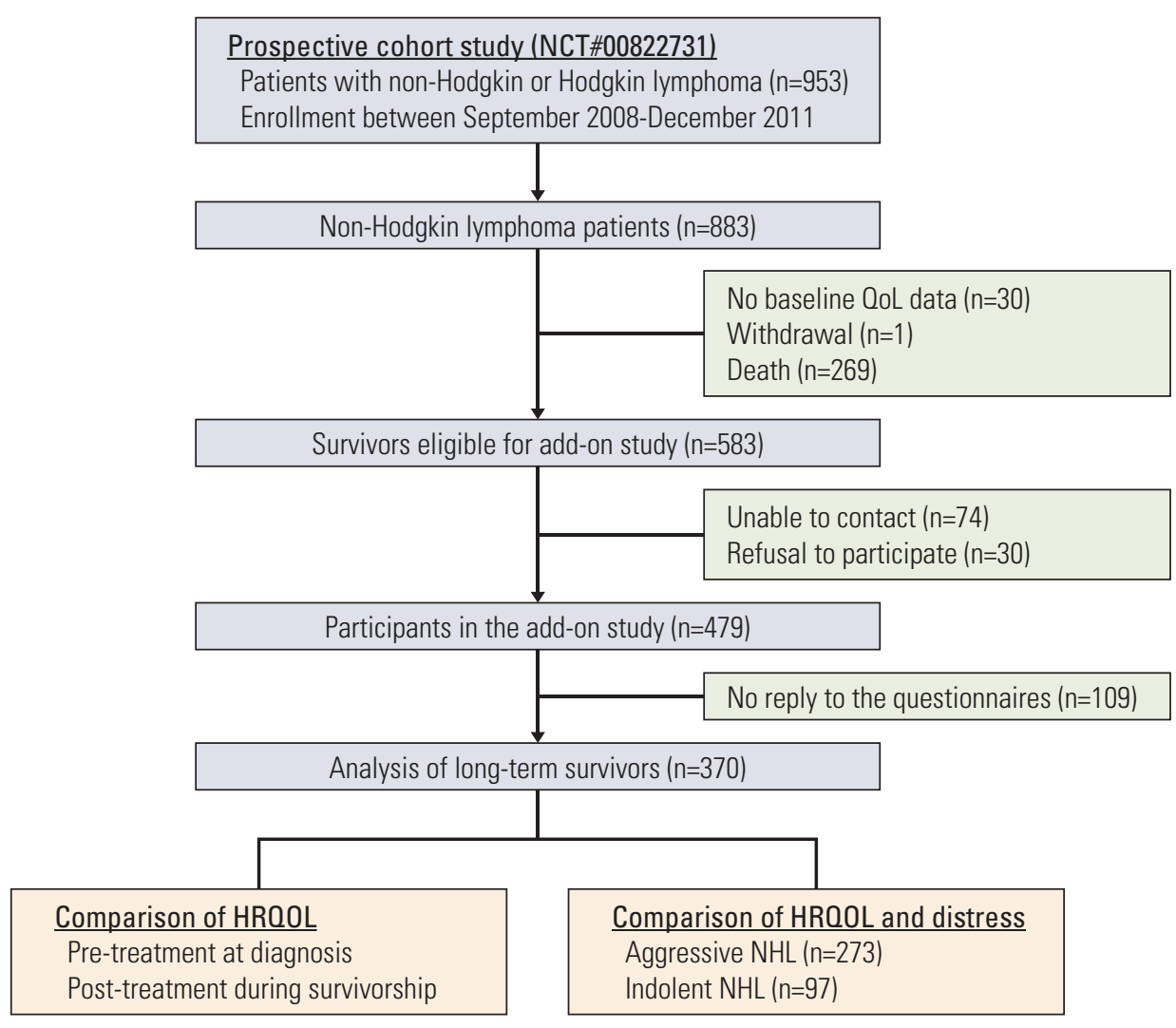

Fig. 1. Consort diagram. QoL, quality of life; HRQOL, health-related quality of life; NHL, non-Hodgkin lymphoma. 
December 2011 with the aim of developing a prediction model for progression and outcomes of patients with lymphoma. The SMC-LCS evaluated all risk factors including diagnostic information, treatment information, and pre-treatment HRQOL at diagnosis. For this add-on study, trained oncology nurses contacted all living SMC-LCS participants who were at least 3 years since diagnosis either by phone or when patients visited the hematology-oncology clinic. Nurses explained the study to participants. Inclusion criteria were patients who were diagnosed with NHL, had baseline HRQOL assessment, and were capable of answering HRQOL questionnaires at the time of the survey. Once patients who were contacted by phone decided to participate in the study, informed consent and survey questionnaires were sent to patients with a return envelope. At out-patient clinics, oncology nurses obtained informed consent, and patients were asked to answer the survey questionnaire before they left the clinic. There were 883 NHL patients enrolled in the SMCLCS. Among them, one patient withdrew informed consent before the baseline assessment, 30 patients did not undergo baseline HRQOL assessment, and 269 patients died before the add-on study. Among 583 eligible participants, we were unable to contact 74 patients, and 30 patients refused to participate in the study. Among 479 survivors who agreed to participate in the study, $109(22.7 \%)$ did not return the survey questionnaire. The final study sample comprised $370 \mathrm{NHL}$ survivors (Fig. 1).

\section{Measurements}

The long-term HRQOL of study participants was evaluated using a validated Korean version of the European Organization for Research and Treatment of Cancer Qualityof-Life Questionnaire Core 30 (EORTC QLQ-C30), the questionnaire used for the original SMC-LCS to assess the quality of life (QoL) of study participants at diagnosis [16]. The EORTC QLQ-C30 is composed of five multi-item functional scales that evaluate physical, role, emotional, cognitive, and social function and one global health status / QoL scale. Three symptom scales measure fatigue, pain, and nausea/vomiting, and six single items assess other symptoms (dyspnea, insomnia, appetite loss, constipation, and diarrhea) and financial difficulties. We scored the EORTC QLQ-C30 items according to a scoring manual, and the data was linearly transformed to yield scores from 0 to 100 [17]. Higher scores indicate a better status in functioning domains but a worse status in symptom domains. The threshold for impaired function and symptoms that negatively affect HRQOL were 66 and 33, respectively [18]. As EORTC QLQ-C30 does not address some important issues of survivorship, such as fear of recurrence and social support, the quality of life in cancer survivors (QOL-CS) questionnaire was included in the study.
The QOL-CS includes 41 items representing four domains (physical, social, psychological, and spiritual well-being) of cancer survivor-specific quality of life [19]. The sum of scores in each domain was used as an outcome, and higher scores indicated a better HRQOL.

After we obtained permission from Dr. Ferrell, who originally developed the measurement, we validated the Korean version of QOL-CS as previously reported [20]. Thus, translation, back-translation, content validity, and pilot tests were performed before the study. Pilot tests with 20 adult survivors of lymphoma showed that Korean survivors were not familiar with the 11-point Likert scale (0 to 10). This scale was one of the main reasons survivors had difficulties answering the questionnaire, so we decided to use a 4-point Likert scale from 1 (not at all) to 4 (very much), in agreement with the original developer (S1 Fig.). With our study participants, the Korean version of QOL-CS was reliable in reporting Cronbach's $\alpha$ for overall CS 0.89 and 0.73 to 0.82 for subdomains [20]. Socio-demographic information including marital status, education, employment, and health behaviors were obtained from survey. Clinical information including age, gender, stage at diagnosis, body mass index, and comorbidities was collected from the electronic medical record.

\section{Statistical analysis}

Subject characteristics and scores of EORTC QLQ-C30 and QOL-CS were summarized using mean \pm standard deviation for continuous variables and number $(\%)$ for categorical variables. Characteristics of each patients group were compared using ANOVA for continuous variables and chi-square tests for categorical variables. For EORCT QLQ-C30, beside reporting transformed score (from 0 to 100), the mean difference of each functioning and symptoms scores from the threshold (66 for functioning and 33 for symptoms) were calculated and presented to present how much worse or better functioning and symptoms NHL survivors experience at different survivorship phase. In addition, to compare changes in quality of life over time, we used linear mixed models. We also tested the homogeneity of slope changes between at diagnosis and during survivorship, and the models were adjusted for age, Eastern Cooperative Oncology Group (ECOG) status and sex. We conducted multivariable linear regression to evaluate the association of three domains of HRQOL in NHL survivors with age at diagnosis (continuous), sex (male or female), disease type (aggressive and indolence), and length of survival (continuous). p-values of $<0.05$ were considered significant, and two-sided tests were used in all calculations. Statistical analyses were performed using STATA ver. 13.0 statistical software (StataCorp LP, College Station, TX) and IBM PASW ver. 18.0 package (SPSS Inc., Chicago, IL). 
Table 1. Sociodemographic and medical characteristics of subjects

\begin{tabular}{|c|c|c|c|c|c|}
\hline Characteristic & $\begin{array}{c}\text { Total } \\
(\mathrm{n}=370)\end{array}$ & $\begin{array}{c}\text { Aggressive NHL } \\
\text { Stage III/IV } \\
(\mathbf{n}=117)\end{array}$ & $\begin{array}{c}\text { Aggressive NHL } \\
\text { Stage I/II } \\
(n=156)\end{array}$ & $\begin{array}{c}\text { Indolent } \\
\text { NHL } \\
(\mathbf{n}=97)\end{array}$ & p-value \\
\hline Female sex, mean $\pm \mathrm{SD}(\mathrm{yr})$ & $164(44.3)$ & $45(38.5)$ & $67(42.9)$ & $52(53.6)$ & 0.08 \\
\hline Age at diagnosis (yr) & $51(18-82)$ & $50(20-82)$ & $53(18-81)$ & $51(18-82)$ & 0.64 \\
\hline Marital status (married) & $302(81.6)$ & $97(82.9)$ & $125(80.1)$ & $80(82.5)$ & 0.80 \\
\hline Education ( $\geq$ college) & $178(48.1)$ & $51(43.6)$ & $78(50.0)$ & $49(50.5)$ & 0.21 \\
\hline Monthly family income $(\geq \$ 4,000)$ & $147(39.7)$ & $41(35.0)$ & $63(40.4)$ & $43(44.3)$ & 0.42 \\
\hline $\begin{array}{l}\text { Current working status } \\
\text { (employed or self-employed) }\end{array}$ & $191(51.6)$ & $55(47.4)$ & $83(53.9)$ & $53(54.6)$ & 0.42 \\
\hline \multicolumn{6}{|l|}{ Smoking status } \\
\hline Never & $225(60.8)$ & $72(61.5)$ & $89(57.1)$ & $63(64.9)$ & 0.13 \\
\hline Past & $122(33.0)$ & $43(36.8)$ & $53(34.0)$ & $27(27.8)$ & \\
\hline Current & $21(5.7)$ & $2(1.7)$ & $12(7.7)$ & $7(7.2)$ & \\
\hline \multicolumn{6}{|l|}{ Drinking status } \\
\hline Never & $166(44.7)$ & $45(38.5)$ & $69(44.2)$ & $52(53.6)$ & 0.03 \\
\hline Past & $66(17.8)$ & $30(25.6)$ & $28(17.9)$ & $8(8.3)$ & \\
\hline Current & $137(37.0)$ & $41(35.0)$ & $59(37.8)$ & $37(38.1)$ & \\
\hline Previous cancer (yes) & $19(5.1)$ & $9(7.7)$ & $6(3.9)$ & $4(4.1)$ & 0.32 \\
\hline Co-morbidity at diagnosis (yes) & $228(61.6)$ & $75(64.1)$ & $97(62.2)$ & $56(57.7)$ & 0.62 \\
\hline ECOG $(\geq 2)$ at diagnosis & $30(8.1)$ & $21(18.0)$ & $8(5.1)$ & $1(1.0)$ & $<0.01$ \\
\hline Time since diagnosis, median (range, yr) & $4.0(1.7-17.4)$ & $3.9(1.7-17.4)$ & $4.1(1.7-6.0)$ & $4.2(1.8-5.8)$ & 0.64 \\
\hline \multicolumn{6}{|l|}{ Treatment history } \\
\hline Chemotherapy (yes) & $318(85.9)$ & $117(100)$ & $155(99.4)$ & $46(47.4)$ & $<0.01$ \\
\hline Radiation therapy (yes) & $84(22.7)$ & $10(8.6)$ & $44(28.2)$ & $30(30.9)$ & $<0.01$ \\
\hline Surgery (yes) & $43(11.6)$ & $9(7.7)$ & $26(16.7)$ & $8(8.3)$ & 0.22 \\
\hline Autologous stem cell transplantation (yes) & $37(10.0)$ & $28(23.9)$ & $9(5.8)$ & 0 & $<0.01$ \\
\hline Relapse after primary treatment & $46(12.4)$ & $22(18.8)$ & $13(8.3)$ & $11(11.3)$ & 0.03 \\
\hline
\end{tabular}

Values are presented as numbers (\%) unless otherwise indicated. NHL, non-Hodgkin lymphoma; SD, standard deviation; ECOG, Eastern Cooperative Oncology Group.

\section{Ethical statement}

This study was approved by the Institutional Review Board of Samsung Medical Center, Seoul, Korea and all patients provided written informed consent prior to study participation (No. 2013-03-051).

\section{Results}

\section{Patients}

The median age of patients was 51 years (range, 18 to 82 years), and $44.5 \%$ were female. The majority was married $(n=302,81.6 \%)$, and $51.6 \%$ of patients were working at the time of survey. Median time from diagnosis to follow-up sur- vey was 4.0 years (range, 1.7 to 17.4 years). In patients with aggressive NHL $(n=273)$, the proportion of DLBCL $(n=186)$ was much higher than that of peripheral T-cell lymphoma $(\mathrm{n}=14)$. Marginal zone lymphoma $(\mathrm{n}=67)$ and follicular lymphoma ( $\mathrm{n}=27)$ accounted for the majority of indolent NHL $(\mathrm{n}=97)$. Among cases of aggressive NHL, $42.9 \%$ were stage III/IV ( $\mathrm{n}=117)$, and $57.1 \%$ were stage I/II $(\mathrm{n}=156)$. None of the socio-demographic characteristics were different between aggressive and indolent NHL (Table 1). ECOG performance status was worse in patients with stage III/IV aggressive NHL than in patients with stage I/II aggressive NHL or indolent NHL. Primary treatment for aggressive NHL was mainly systemic chemotherapy, while $30.9 \%$ of patients with indolent NHL received radiation therapy (Table 1). At the time of analysis, $12.4 \%$ of patients relapsed after their primary treatment, and relapse was more frequent in stage III/IV aggressive NHL (Table 1). 

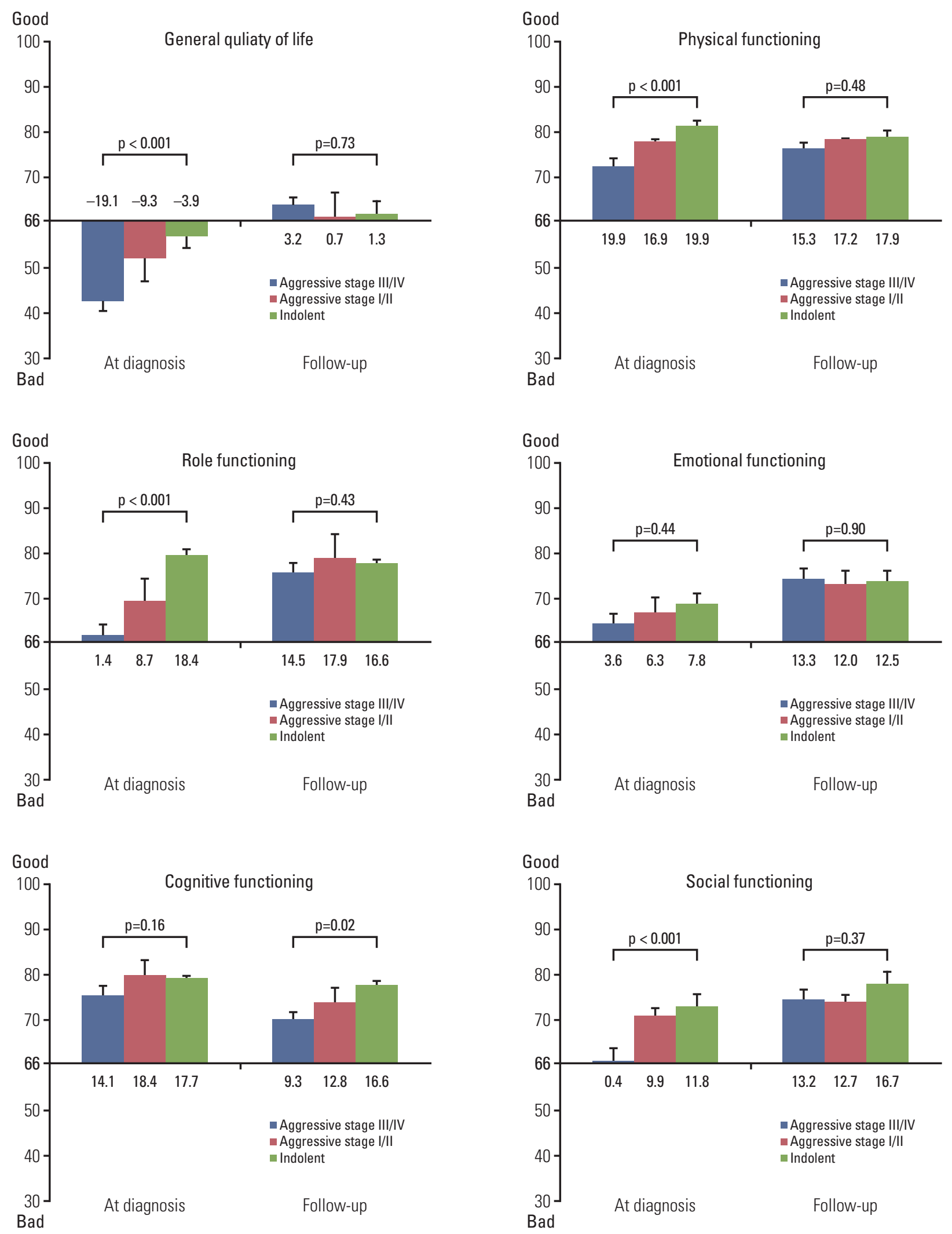

Fig. 2. (A) Comparison of health-related quality of life at diagnosis and during follow-up in aggressive or indolent nonHodgkin lymphoma (NHL). (Continued to the next page) 

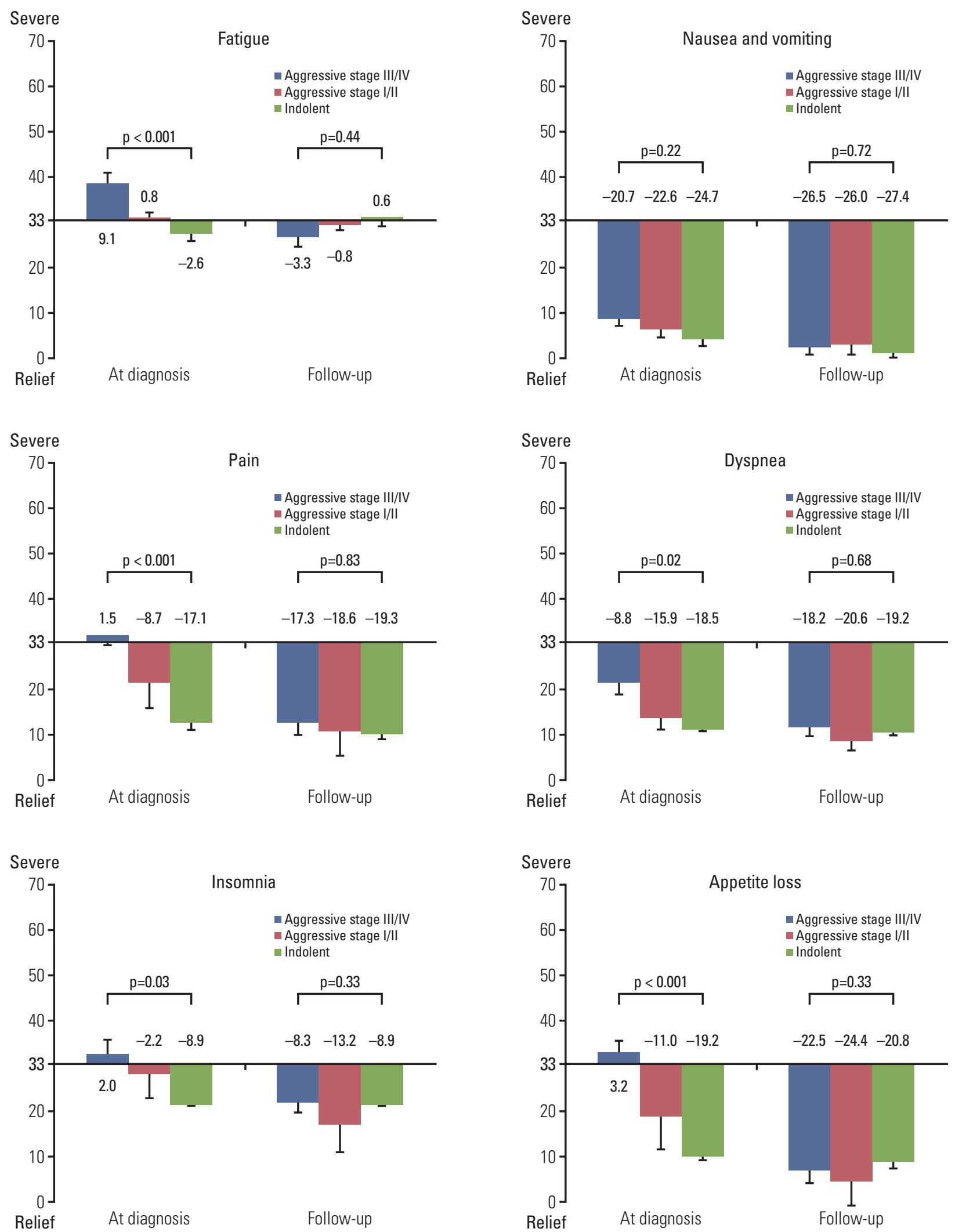

Fig. 2. (Continued from the previous page) (B) Comparison of symptoms at diagnosis and during follow-up in aggressive or indolent NHL. (Continued to the next page) 

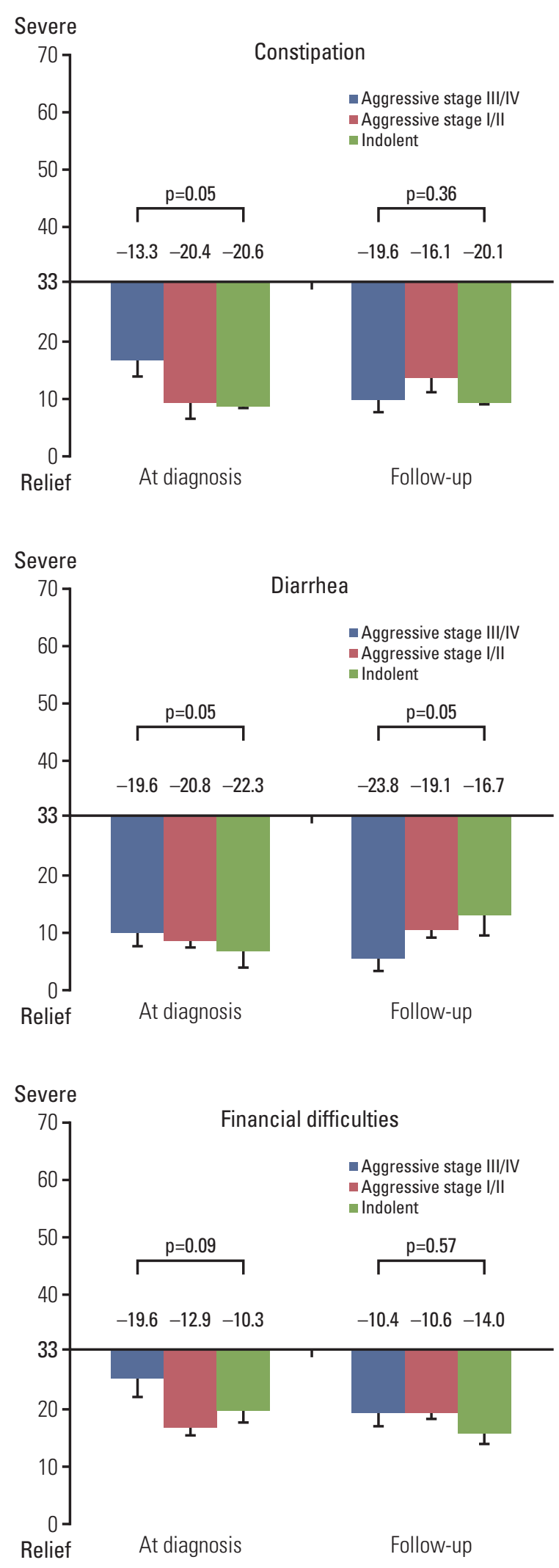

Fig. 2. (Continued from the previous page)

\section{HRQOL at diagnosis and during survivorship}

A comparison of HRQOL at diagnosis shows that patients with stage III/IV aggressive NHL had significantly worse general health status, physical functioning, and role functioning than patients with stage I/ II aggressive or indolent NHL $(\mathrm{p}<0.001)$ (Fig. 2A, S2 Table). However, the follow-up survey showed no difference in HRQOL among NHL survivors regardless of aggressiveness or stage (Fig. 2A, S2 Table). Thus, the follow-up survey showed improvement of EORTC QLQ-C30 symptom scores compared to that of diagnosis scores in survivors of both aggressive and indolent NHL (Fig. 2B, S2 Table). Although survivors of aggressive NHL, especially stage III/IV disease, experienced more serious fatigue, pain, dyspnea, insomnia, and appetite loss than survivors with indolent NHL at the time of diagnosis, they reported improved symptoms at the follow-up survey and their symptom scores improved up to those of indolent NHL. As a result, symptom scores of long-term survivors with NHL were similar for aggressive and indolent NHL at the time of the follow-up survey (Fig. 2B, S2 Table).

\section{Distress and fear of survivors}

The QOL-CS survey showed that more than $60 \%$ of patients experienced distress when they were diagnosed with NHL for the first time, and the degree of distress was not significantly different among patients with aggressive or indolent NHL ( $p=0.98$ ) (Fig. 3). Thus, diagnosis of NHL itself is a significantly stressful event to lymphoma patients regardless of whether the diagnosis is aggressive or indolent NHL. However, patients with aggressive NHL showed more distress about NHL treatment than patients with indolent NHL. This result suggests that patients with aggressive NHL were more concerned about their treatment (Fig. 3). Conversely, survey results on fear of the probability of disease relapse, metastasis, and second malignancy showed no significant differences among patients with aggressive or indolent NHL ( $p>0.05$ ) (Fig. 3). Thus, survivors of NHL were suffering from their fear of relapse and second malignancy, and the degree of fear was not different between survivors with aggressive stage I/ II or III / IV NHL (Fig. 3).

\section{Psychosocial, social, and spiritual well-being of sur- vivors}

Accordingly, 40\%-50\% of survivors were not happy or satisfied with their life. Among negative answers to questionnaires about psychosocial well-being, more than $60 \%$ of survivors were not satisfied with their present ability to concentrate or remember things (Table 2). This impaired sense of psychosocial well-being was found at the time of the fol- 

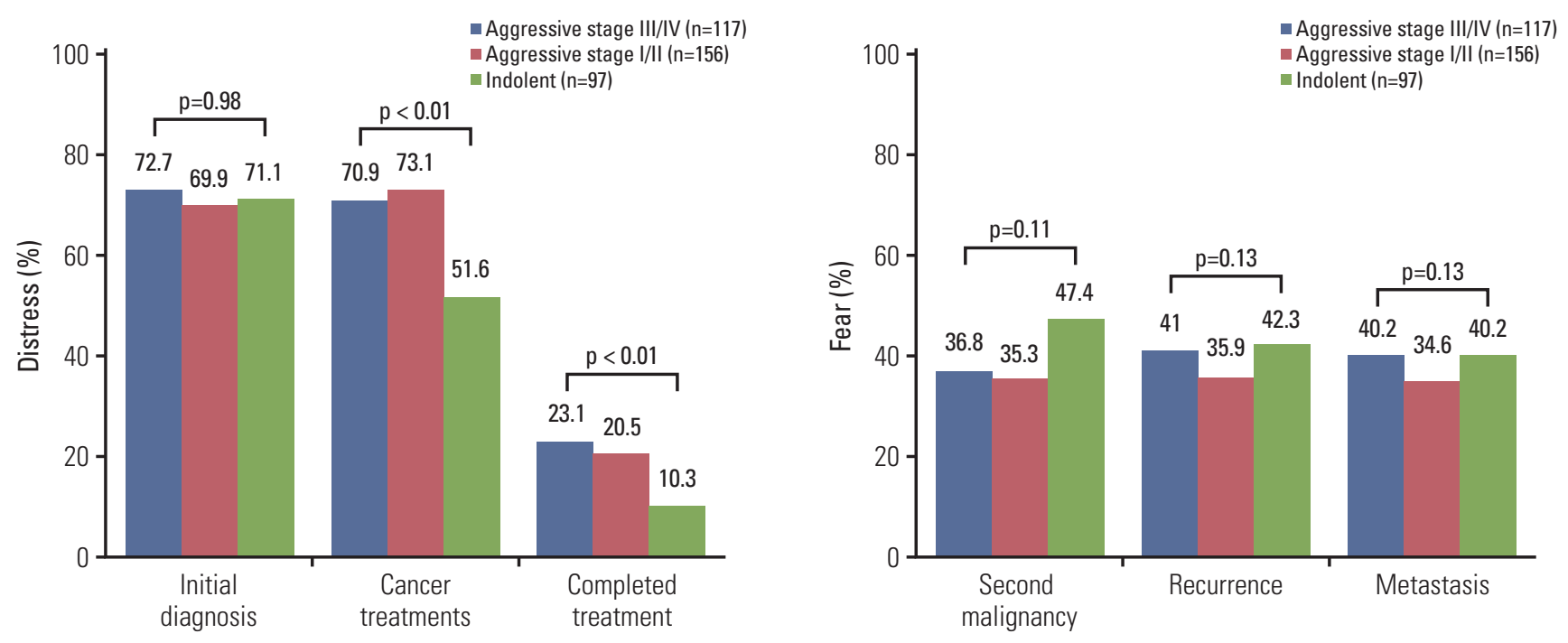

Fig. 3. Distress and fear in aggressive or indolent non-Hodgkin lymphoma.

low-up survey in survivors with aggressive NHL of stage I/II or III/ IV, as well as survivors with indolent NHL (Table 2). In an assessment of social well-being, less than $10 \%$ of survivors reported that their continuing health care interfered with their personal relationships. This result did not differ among aggressive and indolent NHL (Table 2). Activities including employment, home activities, and sexuality were impaired in less than 15\%-20\% of survivors. However, more than $65 \%$ of survivors thought they did not receive sufficient support from others, and more than $15 \%$ survivors had financial burdens incurred as a result of NHL (Table 2). A higher number of survivors with stage III/IV aggressive NHL $(12.8 \%)$ reported isolation, although this was not statistically significant $(p=0.08$ ) (Table 2 ). Answers to questions about spiritual well-being were similar among survivors with aggressive or indolent NHL. In particular, $40 \%$ of survivors did not feel hopeful and reported a lack of life purpose (Table 2). Regardless of stage or aggressiveness, a substantial number of NHL survivors had problems with psychosocial, social, and spiritual well-being, especially, impaired concentration and memory, insufficient support, and lack of life purpose.

\section{Associations between HRQOL at diagnosis and psycho- logical, social, and spiritual well-being during survivor- ship}

We analyzed factors influencing psychosocial, social, and spiritual well-being of long-term survivors under the hypothesis that HRQOL at diagnosis might be associated with impaired well-being during survivorship. Thus, the baseline characteristics and HRQOL data at diagnosis were compared with respect to the aforementioned three main problems of well-being: impaired concentration and memory, insufficient support, and lack of life purpose (Table 3). Demographic and clinical characteristics at diagnosis, including age, performance status, aggressive NHL, treatment, and co-morbidities, were not significantly associated with the above-mentioned problems (Table 3). Instead, patients with financial difficulties (odds ratio [OR], 1.11; 95\% confidence interval [CI], 1.02 to 1.20 ) at diagnosis were more frequently associated with suffering from insufficient support. Patients with a poor general health status and impaired HRQOL at diagnosis were found to have impaired ability to concentrate and impaired memory, and this result was significantly significant (OR, $0.85 ; 95 \%$ CI, 0.77 to 0.93 ) (Table 3). Impaired functioning such as physical, role, and cognitive functioning was significantly associated with problems of concentration and memory. Impaired HRQOL, including function at diagnosis, was also significantly associated with lack of life purpose in longterm survivors (OR, 0.89; 95\% CI, 0.81 to 0.98 ) (Table 3). Long-term survivors with more symptoms represented by higher symptom scores showed a higher incidence of problems in concentration and memory as well as a lack of life purpose (Table 3). When we re-analyzed using domain instead single item as outcome variables, the trend was similar as Table 3 (S3 Table). 


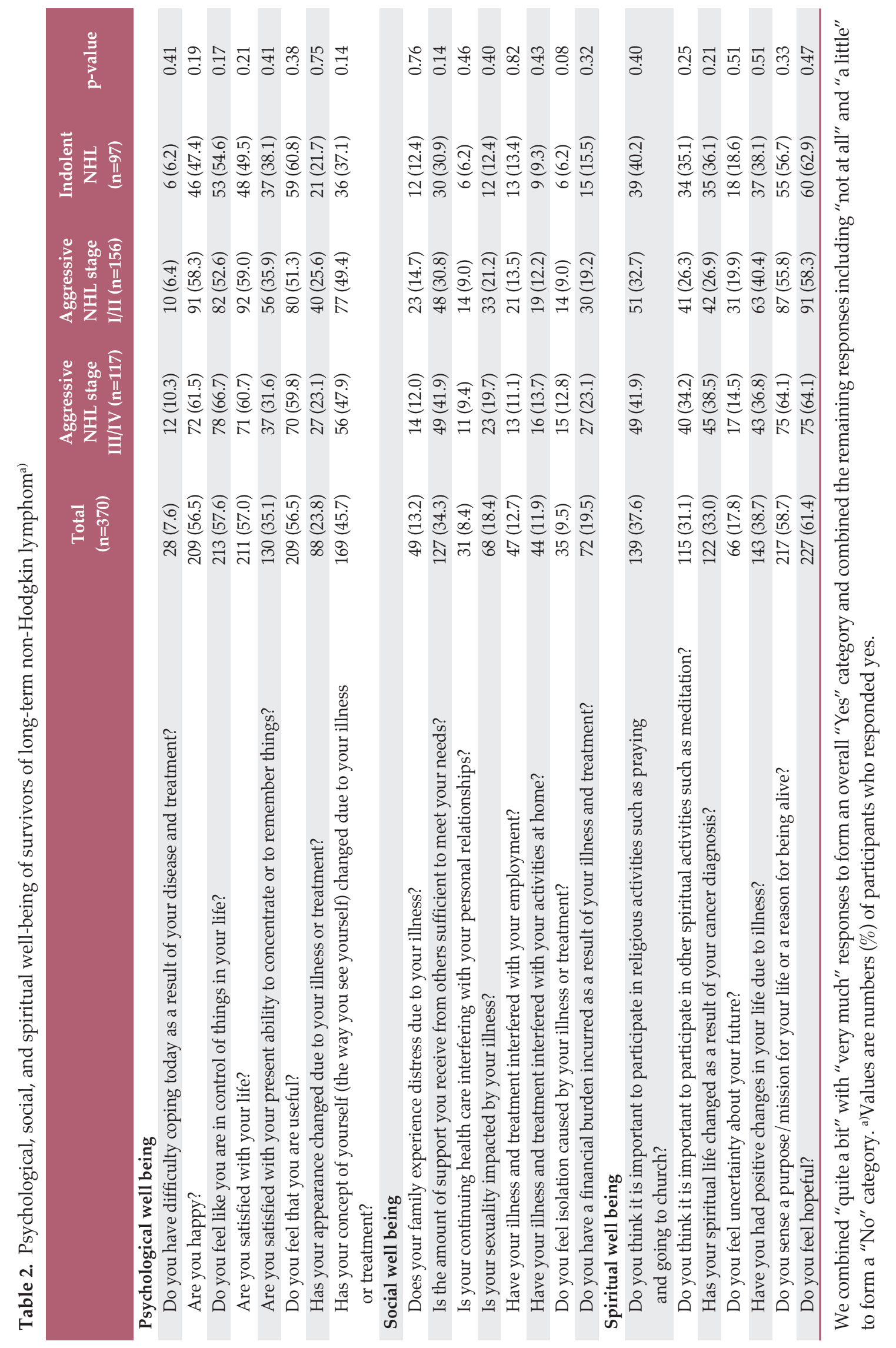


Table 3. Associations between pre-treatment QoL and long-term psychosocial problems in survivors of NHL

\begin{tabular}{|c|c|c|c|}
\hline & \multicolumn{3}{|c|}{ OR $(95 \%$ CI $)$} \\
\hline & $\begin{array}{c}\text { Problem with } \\
\text { concentration/memory }\end{array}$ & $\begin{array}{l}\text { Insufficient } \\
\text { support }\end{array}$ & $\begin{array}{c}\text { Lack of } \\
\text { life purpose }\end{array}$ \\
\hline Female sex & $1.39(0.90-2.17)$ & $0.83(0.57-1.32)$ & $0.79(0.52-1.21)$ \\
\hline Age at diagnosis ( $>50 \mathrm{yr}$ ) & $1.36(0.64-2.85)$ & $1.22(0.58-2.55)$ & $1.59(0.76-3.27)$ \\
\hline History of cancer (yes) & $1.47(0.50-4.28)$ & $0.59(0.22-1.57)$ & $2.01(0.76-5.29)$ \\
\hline Relapse (yes) & $1.70(0.93-3.51)$ & $1.24(0.62-2.48)$ & $1.58(0.83-2.99)$ \\
\hline Type of diagnosis (aggressive type) & $1.22(0.74-2.00)$ & $0.75(0.46-1.24)$ & $0.88(0.54-1.42)$ \\
\hline \multicolumn{4}{|l|}{ History of treatment } \\
\hline Chemotherapy (yes) & $2.33(0.99-5.45)$ & $1.67(0.70-3.96)$ & $1.81(0.79-4.11)$ \\
\hline Radiation therapy (yes) & $0.83(0.49-1.39)$ & $0.60(0.36-1.01)$ & $0.63(0.37-1.06)$ \\
\hline Baseline ECOG $(\geq 2)$ & $2.24(0.88-5.70)$ & $0.91(0.41-2.03)$ & $1.82(0.83-3.92)$ \\
\hline Length of survival at second survey (yr) & $1.03(0.84-1.27)$ & $1.18(0.97-1.44)$ & $0.84(0.68-1.03)$ \\
\hline Co-morbidity & $1.42(0.90-2.23)$ & $1.36(0.86-2.14)$ & $1.54(0.98-2.40)$ \\
\hline General health status/QoL & $0.85(0.77-0.93)^{a)}$ & $0.91(0.83-1.00)$ & $\left.0.89(0.81-0.98)^{a}\right)$ \\
\hline \multicolumn{4}{|l|}{ Functioning } \\
\hline Physical functioning & $0.73(0.62-0.85)^{a)}$ & $0.94(0.82-1.07)$ & $0.81(0.72-0.92)^{a)}$ \\
\hline Role functioning & $0.85(0.78-0.93)^{\mathrm{a})}$ & $0.96(0.89-1.04)$ & $\left.0.92(0.85-0.99)^{a}\right)$ \\
\hline Emotional functioning & $0.88(0.80-0.97)^{a)}$ & $0.98(0.90-1.08)$ & $0.96(0.88-1.05)$ \\
\hline Cognitive functioning & $0.75(0.66-0.86)^{a)}$ & $0.94(0.84-1.06)$ & $\left.0.89(0.79-0.99)^{a}\right)$ \\
\hline Social functioning & $0.90(0.83-0.98)^{a)}$ & $1.00(0.93-1.08)$ & $0.96(0.89-1.03)$ \\
\hline \multicolumn{4}{|l|}{ Symptoms } \\
\hline Fatigue & $1.23(1.12-1.36)^{a)}$ & $1.02(0.93-1.11)$ & $1.08(1.00-1.18)^{a)}$ \\
\hline Nausea and vomiting & $1.21(1.04-1.41)^{\mathrm{a})}$ & $0.99(0.88-1.14)$ & $1.05(0.92-1.20)$ \\
\hline Pain & $1.09(0.99-1.19)$ & $1.00(0.93-1.09)$ & $1.04(0.96-1.12)$ \\
\hline Dyspnea & $1.12(1.02-1.23)^{\mathrm{a})}$ & $1.04(0.96-1.14)$ & $1.04(0.96-1.12)$ \\
\hline Insomnia & $1.11(1.03-1.20)^{a)}$ & $0.99(0.92-1.07)$ & $1.08(1.01-1.16)^{a)}$ \\
\hline Appetite loss & $1.08(1.00-1.16)^{a)}$ & $0.99(0.93-1.07)$ & $1.09(1.02-1.17)^{a)}$ \\
\hline Constipation & $1.02(0.94-1.11)$ & $1.00(0.92-1.09)$ & $1.02(0.94-1.10)$ \\
\hline Diarrhea & $1.15(1.03-1.30)^{\mathrm{a})}$ & $1.06(0.96-1.17)$ & $1.05(0.96-1.15)$ \\
\hline Financial difficulties & $1.14(1.05-1.24)^{a)}$ & $1.11(1.02-1.20)^{\mathrm{a})}$ & $1.05(0.98-1.13)$ \\
\hline
\end{tabular}

OR and 95\% CI for \% of problems defined by "not at all" and "a little" were calculated using logistic regression adjusted for age at diagnosis (continuous), sex (male or female), disease type (aggressive and indolence), and length of survival (continuous). QoL, quality of life; NHL, non-Hodgkin lymphoma; OR, odds ratio; CI, confidence interval; ECOG, Eastern Cooper-

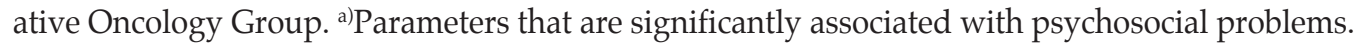

\section{Discussion}

This study evaluated the HRQOL of long-term survivors during survivorship. The study population included patients diagnosed with aggressive or indolent NHL. Because HRQOL at diagnosis was evaluated when patients were registered in the prospective cohort study, the HRQOL of longterm survivors during survivorship was compared with that of patients at diagnosis. The comparison of HRQOL at diagnosis showed that patients with stage III/IV aggressive NHL had significantly worse HRQOL than those with stage I/II aggressive or indolent NHL (Fig. 2A). However, this differ- ence was lost in long-term survivors because the EORTC QLQ-C30 symptom scores became similar between aggressive and indolent NHL at the time of the follow-up survey (Fig. 2B). This result implies that patients with aggressive NHL, even stage III/IV, can recover without prolonged deterioration of HRQOL. This result is consistent with a previous study reporting similar HRQOL between survivors of indolent and aggressive NHL [21]. Additionally, we found that NHL survivors reported a much lower HRQOL than that of the general population. A cross sectional study, which used the EORTC QLQ-C30 to measure the HRQOL of in the general population, demonstrated that the physical, role, emotional, cognitive, and social function scores of the general 
population were on average $85.1,88.0,83.4,86.5$, and 92.5 , respectively [10] which were much higher than the scores of the indolent NHL survivors we measured. In our study, we found that functioning and symptoms at diagnosis were significantly associated with long-term HRQOL both in aggressive and indolent NHL survivors. Given that HRQOL at diagnosis could be one of prognostic predictors in NHL patients [22], the assessment of HRQOL at the time of diagnosis as well as the appropriate management based on the follow-up assessment of HRQOL during survivorship may be necessary in both aggressive and indolent NHL patients [23].

The majority of long-term survivors in this study reported fear of relapse or second malignancy as their most distressing problem, regardless of NHL aggressiveness or stage. Indolent NHL survivors experienced the same level of fear of relapse or second malignancy as stage III/IV aggressive NHL survivors. These results are consistent with a previous review of cancer survivors reporting that fear of relapse could be the primary concern of cancer survivors regardless of cancer type, stage at diagnosis, disease progression, or length of survivorship [24]. Thus, cancer survivors constantly live anxiously about their recurrence potential, especially when they have any symptoms such as fatigue, new pains, and feeling of weakness [25,26]. Therefore, adequate education about the probability of relapse and second malignancy is required. Indeed, a previous Dutch population-based study has shown more information about the disease and post-treatment care might improve stress in patients with lymphoma [27]. For effective education for patients as well as survivors, adequate doctor-patient relationship is important because it is at the crux of cancer care. The National Cancer Institute stated that patient-centered care should be based on mutual trust, respect, and commitment, and care could be facilitated by communication [28]. Nevertheless, physicians still focus on detecting relapses through regular imaging rather than managing HRQOL-associated problems in clinical practice. Indeed, many survivors adhere to surveillance testing, and feel reassured when they get negative results. Physicians can also be reassured by negative test results and be more confident in reassuring the patients. However, lymphoma is a basically curable disorder by chemotherapy and the majority of relapses occur within 2 years after the completion of treatment. Thus, the probability of relapse in longterm survivors of lymphoma is low and surveillance imaging was reported to lack clinical utility in lymphoma patients $[29,30]$. Actually, routine imaging such as surveillance computed tomography scans could be a source of anxiety and fear of relapse in lymphoma survivors without improving post-treatment survival [31,32]. Therefore, proper monitoring and symptom management needs to be embedded in the routine practice of long-term survivor care because psycho- logical interventions such as Mindfulness Based Stress Reduction, Mindfulness Based Cognitive Therapy, and Acceptance and Commitment Therapy might be helpful to reduce fears of relapse [33,34].

This study also assessed the psychosocial, social, and spiritual well-being of long-term survivors during survivorship. Three main problems were identified, including impaired memory and ability to concentrate, insufficient support, and lack of life purpose (Table 2). Thus, regardless of aggressiveness and stage, a substantial number of long-term NHL survivors seemed to suffer from those problems. Impaired memory and ability to concentrate and lack of life purpose were significantly associated with HRQOL at diagnosis. Patients with poor HRQOL might suffer more from impaired psychosocial and spiritual well-being although their HRQOL and symptoms improve during survivorship. These findings suggest that patients with poor HRQOL at diagnosis should be more appropriately managed to improve their psychosocial and spiritual well-being. In an assessment of social wellbeing, more than $65 \%$ of survivors thought they did not receive sufficient support from others, and more than $15 \%$ of survivors incurred financial burdens as a result of NHL (Table 2). This is consistent with a previous study reporting that cancer patients continuously need supportive care [35]. Psychosocial care for cancer survivors after completing medical treatment has been suggested because cancer survivors are vulnerable to feeling a loss of safety [36]. In our study, patients with financial difficulties at diagnosis more frequently reported that they had a lack of sufficient supportive care (Table 3). Thus, intervention for patients with financial problems should be considered to improve social well-being during survivorship.

However, this study has several limitations. First, we only analyzed subjects who participated to the survey in this study. However, $22.7 \%$ of subjects did not return the survey questionnaire among 479 survivors who agreed to participate in the study although this response rate was similar to that of other studies [9]. This means this study might be influenced by selection bias because it would be possible that subjects with good HRQOL might more respond than subjects with impaired HRQOL and psychosocial problems. Thus, HRQOL of the NHL survivors in our study would be better than those in actual survivors. Second, the results of our study were absolutely based on self-reported outcomes of subjects. Thus, the results might be influenced by individual sensitivity. However, we used the validated and standardized instruments to assess study outcomes to overcome the limitation of self-reported outcome-based study. Third, when we evaluated the association of pre-treatment HRQOL with long-term psychosocial problems, we only adjusted for age, sex, and diagnosis due to relatively small sample size. Thus, further study with larger study population should be 
done to identify the risk factors for long-term survivors of NHL.

In conclusion, long-term survivors of NHL reported improved HRQOL during survivorship. Thus, the HRQOL of survivors of aggressive NHL improved to a similar level to that of survivors of indolent NHL. However, most of the long-term NHL survivors still live with fear of relapse, insufficient social support, and lack a purpose in life. Thus, adequate education and guidance for long-term survivors are warranted and recommended during survivorship.

\section{Electronic Supplementary Material}

Supplementary materials are available at Cancer Research and Treatment website (http:// www.e-crt.org).

\section{Conflicts of Interest}

Conflict of interest relevant to this article was not reported.

\section{Acknowledgments}

This study was supported by a grant from the 20-20 Project of Samsung Medical Center.

\section{References}

1. Howlader N, Krapcho M, Miller D, Bishop K, Altekruse SF, Kosary CL, et al. SEER cancer statistics review, 1975-2013. Bethesda, MD: National Cancer Institute; 2016.

2. Jung KW, Won YJ, Oh CM, Kong HJ, Lee DH, Lee KH, et al. Cancer statistics in Korea: incidence, mortality, survival, and prevalence in 2014. Cancer Res Treat. 2017;49:292-305.

3. Molina A. A decade of rituximab: improving survival outcomes in non-Hodgkin's lymphoma. Annu Rev Med. 2008;59: 237-50.

4. Khan N, Cheson BD. PTCL therapies: a review of treatment and outline of novel therapies. Am J Ther. 2013;20:524-33.

5. Safdari Y, Ahmadzadeh V, Farajnia S. CD20-targeting in B-cell malignancies: novel prospects for antibodies and combination therapies. Invest New Drugs. 2016;34:497-512.

6. Pfreundschuh M, Kuhnt E, Trumper L, Osterborg A, Trneny $\mathrm{M}$, Shepherd L, et al. CHOP-like chemotherapy with or without rituximab in young patients with good-prognosis diffuse large-B-cell lymphoma: 6-year results of an open-label randomised study of the MabThera International Trial (MInT) Group. Lancet Oncol. 2011;12:1013-22.

7. Oerlemans S, Mols F, Nijziel MR, Lybeert M, van de PollFranse LV. The impact of treatment, socio-demographic and clinical characteristics on health-related quality of life among Hodgkin's and non-Hodgkin's lymphoma survivors: a systematic review. Ann Hematol. 2011;90:993-1004.

8. Oerlemans S, Mols F, Issa DE, Pruijt JH, Peters WG, Lybeert $\mathrm{M}$, et al. A high level of fatigue among long-term survivors of non-Hodgkin's lymphoma: results from the longitudinal population-based PROFILES registry in the south of the Netherlands. Haematologica. 2013;98:479-86.

9. Smith SK, Mayer DK, Zimmerman S, Williams CS, Benecha H, Ganz PA, et al. Quality of life among long-term survivors of non-Hodgkin lymphoma: a follow-up study. J Clin Oncol. 2013;31:272-9.

10. Kim SH, Kim IR, Kim SH, Lee S, Ok O, Kim WS, et al. Health- related quality of life in Korean lymphoma survivors compared with the general population. Ann Hematol. 2014;93: 1531-40.

11. Arden-Close E, Pacey A, Eiser C. Health-related quality of life in survivors of lymphoma: a systematic review and methodological critique. Leuk Lymphoma. 2010;51:628-40.

12. Mols F, Aaronson NK, Vingerhoets AJ, Coebergh JW, Vreugdenhil G, Lybeert ML, et al. Quality of life among long-term non-Hodgkin lymphoma survivors: a population-based study. Cancer. 2007;109:1659-67.

13. Smith SK, Zimmerman S, Williams CS, Zebrack BJ. Health status and quality of life among non-Hodgkin lymphoma survivors. Cancer. 2009;115:3312-23.

14. Jensen RE, Arora NK, Bellizzi KM, Rowland JH, Hamilton AS, Aziz NM, et al. Health-related quality of life among survivors of aggressive non-Hodgkin lymphoma. Cancer. 2013;119:67280.

15. Kim SH, Lee S, Kim SH, Ok ON, Kim IR, Choi E, et al. Unmet needs of non-Hodgkin lymphoma survivors in Korea: prevalence, correlates, and associations with health-related quality of life. Psychooncology. 2017;26:330-6.

16. Yun YH, Park YS, Lee ES, Bang SM, Heo DS, Park SY, et al. Validation of the Korean version of the EORTC QLQ-C30. Qual Life Res. 2004;13:863-8.

17. Fayers PM, Aaronson NK, Bjordal K, Groenvold M, Curran D, Bottomley A. The EORTC QLQ-C30 scoring manual. 3rd ed. Brussels: European Organization for Research and Treatment of Cancer; 2001.

18. Stromgren AS, Goldschmidt D, Groenvold M, Petersen MA, Jensen PT, Pedersen L, et al. Self-assessment in cancer patients referred to palliative care: a study of feasibility and symptom epidemiology. Cancer. 2002;94:512-20.

19. Ferrell BR, Dow KH, Grant M. Measurement of the quality of life in cancer survivors. Qual Life Res. 1995;4:523-31.

20. Cho J, Kang D, Kim IR, Kim WS, Ferrell B, Kim SJ. Validation 
of the Korean version of the Quality of Life-Cancer Survivors (QOL-CS-K) questionnaire in lymphoma survivors. Cancer Res Treat. 2018;50:204-11.

21. Blaes AH, Ma L, Zhang Y, Peterson BA. Quality of life appears similar between survivors of indolent and aggressive nonHodgkin lymphoma. Leuk Lymphoma. 2011;52:2105-10.

22. Jung HA, Park S, Cho JH, Kim S, Ko YH, Kim SJ, et al. Prognostic relevance of pretreatment quality of life in diffuse large B-cell lymphoma patients treated with rituximab-CHOP: results from a prospective cohort study. Ann Hematol. 2012;91:1747-56.

23. Mertz BG, Dunn-Henriksen AK, Kroman N, Johansen C, Andersen KG, Andersson M, et al. The effects of individually tailored nurse navigation for patients with newly diagnosed breast cancer: a randomized pilot study. Acta Oncol. 2017;56: 1682-9.

24. Crist JV, Grunfeld EA. Factors reported to influence fear of recurrence in cancer patients: a systematic review. Psychooncology. 2013;22:978-86.

25. Lebel S, Maheu C, Lefebvre M, Secord S, Courbasson C, Singh $\mathrm{M}$, et al. Addressing fear of cancer recurrence among women with cancer: a feasibility and preliminary outcome study. J Cancer Surviv. 2014;8:485-96.

26. Gill KM, Mishel M, Belyea M, Germino B, Germino LS, Porter $\mathrm{L}$, et al. Triggers of uncertainty about recurrence and longterm treatment side effects in older African American and Caucasian breast cancer survivors. Oncol Nurs Forum. 2004;31:633-9.

27. Oerlemans S, Husson O, Mols F, Poortmans P, Roerdink H, Daniels LA, et al. Perceived information provision and satisfaction among lymphoma and multiple myeloma survivors: results from a Dutch population-based study. Ann Hematol. 2012;91:1587-95.
28. Epstein RM, Street RL. Patient-centered communication in cancer care: promoting healing and reducing suffering. Bethesda, MD: National Cancer Institute; 2007.

29. Phillips T, Mercer J. Surveillance scans in lymphoma: friend or foe? Curr Treat Options Oncol. 2017;18:10.

30. Guidot DM, Switchenko JM, Nastoupil LJ, Koff JL, Blum KA, Maly J, et al. Surveillance imaging in mantle cell lymphoma in first remission lacks clinical utility. Leuk Lymphoma. 2018;59:888-95.

31. Thompson CA, Charlson ME, Schenkein E, Wells MT, Furman RR, Elstrom R, et al. Surveillance CT scans are a source of anxiety and fear of recurrence in long-term lymphoma survivors. Ann Oncol. 2010;21:2262-6.

32. El-Galaly TC, Jakobsen LH, Hutchings M, de Nully Brown P, Nilsson-Ehle H, Szekely E, et al. Routine imaging for diffuse large B-cell lymphoma in first complete remission does not improve post-treatment survival: a Danish-Swedish population-based study. J Clin Oncol. 2015;33:3993-8.

33. Abernethy AP, Etheredge LM, Ganz PA, Wallace P, German RR, Neti C, et al. Rapid-learning system for cancer care. J Clin Oncol. 2010;28:4268-74.

34. Gil KM, Mishel MH, Belyea M, Germino B, Porter LS, Clayton $\mathrm{M}$. Benefits of the uncertainty management intervention for African American and White older breast cancer survivors: 20-month outcomes. Int J Behav Med. 2006;13:286-94.

35. Molassiotis A, Wilson B, Blair S, Howe T, Cavet J. Unmet supportive care needs, psychological well-being and quality of life in patients living with multiple myeloma and their partners. Psychooncology. 2011;20:88-97.

36. Stanton AL. What happens now? Psychosocial care for cancer survivors after medical treatment completion. J Clin Oncol. 2012;30:1215-20. 